\title{
Non-convulsive status epilepticus: causes, treatment, and outcome in 65 patients
}

\author{
Frans B Scholtes, Willy O Renier, Harry Meinardi
}

\begin{abstract}
The incidence of non-convulsive status epilepticus (NCSE) in The Netherlands is not known. Files of admissions in the years 1980-7 were studied from 40 adult patients (older than 15 years) with complex partial status epilepticus (CPSE) and 25 with absence status epilepticus (ASE). The clinical presentation sometimes made distinction between CPSE and ASE possible. Focal clinical signs were more frequent in CPSE; a fluctuating level of consciousness was more often present in ASE. All patients, but one, with ASE and most patients with CPSE (28) were known to have had previous epilepsy. Outcome in ASE was good in all. Outcome in CPSE depended on the underlying cause and quality of treatment. In three patients inadequate treatment probably contributed to morbidity.
\end{abstract}

(F Neurol Neurosurg Psychiatry 1996;61:93-95)

Keywords: non-convulsive status epilepticus; absence status epilepticus; complex partial status epilepticus; treatment; outcome

Non-convulsive status epilepticus (NCSE) is characterised by a clouding of consciousness, confusion, automatisms, and amnesia, with specific EEG abnormalities in accord with the type of NCSE.

In adult patients NCSE is divided in two main groups, generalised non-convulsive SE and complex partial SE, which, because of their clinical similarities, have been discussed together by most authors.

Generalised non-convulsive SE includes absence SE (ASE) and atypical absence SE. The first description of ASE was first described by Lennox in 1945; since then several reports have appeared which describe absence SE under a variety of names: Absence $S E$, petit mal $\mathrm{SE}$, epilepsia minor continua, spike wave stupor, and prolonged petit mal automatisms. At the moment absence SE is the most often used term to describe a prolonged confusional state of varying severity, with a fluctuating level of consciousness together with generalised paroxysmal activity on the EEG..$^{1-3}$ Therefore in this paper ASE is equivalent to generalised nonconvulsive SE.

The first description of complex partial status epilepticus (CPSE) was in 1956 by Gastaut. ${ }^{4}$ The clinical presentation has been described extensively by others. ${ }^{5-10}$

\section{NCSE in The Netherlands}

Our study concerns patients admitted to hospital. We tried to find out how the diagnosis of a particular type of NCSE had been made and we were especially interested in clinical presentation, management, and outcome. Some patients with NCSE will not be diagnosed, or will not be sent to a hospital if the NCSE subsided spontaneously. Others are known to have had previous epilepsy or previous episodes of NCSE and immediate admission to hospital is not always required.

\section{Methods}

The population of The Netherlands is about 15 million. The incidence of patients with NCSE in The Netherlands is not known. We asked for the cooperation of neurologists in 50 medical centres in The Netherlands. Fourteen, from all parts of the country, agreed to cooperate, information from two was inadequate. Also two of the three epilepsy centres were included.

The study was limited to NCSE in patients over 15 years of age admitted to hospital during 1980-7. Only patients with a minimum seizure duration of 30 minutes were included. Outcome is the condition at discharge from the hospital. Morbidity is all new neurological signs occurring because of the status epilepticus. Therapy was considered insufficient when an insufficient dose had been administered, when the route of administration was wrong (for example, diazepam given intramuscularly), when unnecessary delay was present (for instance waiting for more than one hour after diazepam injection, while seizures continued, before giving a second agent), when mechanical ventilation was not started despite signs of respiratory insufficiency or the presence of various medical complications, or when EEG monitoring was not done in patients treated with antiepileptic drugs together with curarisation. 


\section{Results}

We found 65 admissions for NCSE, 40 with CPSE, and 25 with ASE in 12 hospitals and two epilepsy centres in The Netherlands (table).

\section{ABSENCE STATUS EPILEPTICUS}

There were 25 admissions because of ASE in 18 patients. One female patient of 72 had six admissions and two patients had two admissions each. Two patients with ASE were mentally retarded; the EEG showed continuous spike slow wave activity.

The clinical presentation of ASE was characterised by a state of altered consciousness. Some seemed alert, but responsiveness was impaired. Others behaved strangely, were laughing without obvious reason, and showed myoclonic jerking of eyelids. Some showed environmentally induced or spontaneous automatisms. A few were very drowsy and did not speak, others were withdrawn and showed stereotypic automatisms such as smiling and head nodding. In eight patients a state of confusion with fluctuating level of consciousness was present. Two patients were incontinent of urine, both were very drowsy. One patient wandered around and showed myoclonic jerks especially in the arms. The diagnosis of ASE was confirmed in 21 patients by EEG.

Precipitating factors of ASE in the 24 patients with previous epilepsy consisted of antiepileptic drug withdrawal or non-compliance (10), systemic infection (one), and stress (one); in 11 patients no cause could be found. One patient exhibited several periods of ASE during one admission, each recurring after defecation. The cause of ASE in one patient without previous epilepsy remained unknown.

Outcome in ASE was good in all patients. Because of this, duration of ASE could not be related to outcome. Duration was less than two hours in eight patients, including one with atypical ASE; between two and 20 hours in 10, 24 hours in four, 48 hours in two (including the other patient with atypical ASE), and 14 days in one patient. The quality of treatment was inadequate in three patients but outcome was good all the same.

\section{COMPLEX PARTIAL STATUS EPILEPTICUS}

There were 40 admissions for CPSE in 37 patients; three male patients had two admissions each and all three had previous epilepsy. CPSE followed one or more tonic-clonic seizures in 10 patients, a tonic-clonic seizure occurred during CPSE in one patient and ended CPSE in two patients.

The clinical presentation of CPSE was characterised by confusion and slowness in response, together with stereotypic and/or com-

Distribution of age and sex in patients with CPSE and ASE

\begin{tabular}{|c|c|c|c|c|c|c|}
\hline & \multicolumn{2}{|c|}{ Age $15-30$} & \multicolumn{2}{|c|}{ Age $30-50$} & \multicolumn{2}{|c|}{$A g e>50$} \\
\hline & Male & Female & Male & Female & Male & Female \\
\hline \multicolumn{7}{|l|}{ CPSE: } \\
\hline Previous epilepsy (28) & 9 & 3 & 5 & 3 & 5 & 3 \\
\hline No previous epilepsy (12) & 0 & 1 & 1 & 2 & 3 & 5 \\
\hline \multicolumn{7}{|l|}{ ASE: } \\
\hline Previous epilepsy (24) & 5 & 4 & 0 & 1 & 4 & 10 \\
\hline No previous epilepsy (1) & 1 & 0 & 0 & 0 & 0 & 0 \\
\hline
\end{tabular}

plex automatisms. Focal clinical signs, such as clonic jerking of $1 \mathrm{arm}$, were present in almost half of the patients, whereas tonic deviation of eyes and head was present in another $15 \%$. About $50 \%$ of the patients showed a succession of complex partial seizures; between the seizures the patients remained confused or unresponsive. Others remained in a continuous state of confusion and were either withdrawn without initiative and hardly responsive, or seemed restless with various automatisms, such as lipsmacking and picking at clothes or objects. Two seemed to have hallucinations. Staring was seen in only two patients.

In patients with previous epilepsy, problems with treatment such as non-compliance were the most frequent precipitating factor (12 patients). On two occasions systemic infection, on one occasion a stroke, and on another stress triggered the CPSE. In 12 patients the cause remained unknown. In patients without previous epilepsy various acute symptomatic causes were present: stroke (two), brain tumour (two), pneumococcal meningo-encephalitis (one), carcinomatous meningitis and lung cancer (one), digoxin intoxication (one), pneumococcal pneumonia (one), and a case without a clear cause. Three patients had remote causes of epilepsy-multiple sclerosis, global cerebral atrophy and chronic dialysis with aluminium encephalopathy. Outcome in patients with previous epilepsy was good in all but one, a 78 year old man who had successful treatment stopping his CPSE but died later because of aspiration pneumonia, acquired during the status epilepticus.

In patients without previous epilepsy six had sequelae after CPSE: paresis because of underlying cause (two), persistent cognitive disturbances because of underlying (one) and unknown cause (one), and persistent impaired consciousness of unknown cause (one). The CPSE itself caused morbidity in a 74 year old woman who developed CPSE lasting 24 hours after digoxin intoxication; she was confused with jerking of the right arm, alternating with short periods of staring. After recovery from CPSE this patient exhibited word finding problems and a memory deficit. Patients with morbidity were, except for one (38 years), older than 60 . In patients without previous epilepsy one patient of 67 died because of the underlying cause (lung cancer and carcinomatous meningitis).

Duration of CPSE was less than two hours in 17, between two and four hours in nine, between four and 12 hours in five, one to three days in seven patients, four days in one, and 16 days in another patient. When the underlying cause was responsible for morbidity (three patients), the damage was present at the start of CPSE; in two of these three patients duration of CPSE was less than two hours. Notwithstanding a duration of CPSE from 20 hours to 16 days all patients except one had a good outcome.

\section{Discussion}

The medical literature on NCSE is extensive but sometimes confusing. Even some patients 
with previous partial epilepsy have been described as having ASE instead of CPSE. ${ }^{3511}$ Others draw general conclusions about patients with NCSE, although different types of NCSE are present. ${ }^{312}$

Most admissions in our study were for CPSE, which confirms the conclusion of Tomson $e t a l^{6}$ that CPSE is more frequent than previously reported. This may be caused by the fact that the ICD-9 did not include CPSE.

Age distribution in patients of CPSE showed no preference in patients with previous epilepsy, but in patients without previous epilepsy most patients were older than 50 years of age.

Morbidity after CPSE is often caused by the aetiology such as a cerebrovascular abnormality or neoplasm, less often by the continuing discharges themselves. ${ }^{1314}$ In our CPSE group inadequate treatment may well have contributed to a worse outcome in three patients.

In ASE all but one patient had idiopathic generalised epilepsy. On average, we did not find a female preference, although women outnumbered men in the elderly age group. Although outcome in ASE was good in all patients, several had had inadequate treatment.

While examining the patient records in the participating hospitals we were struck by the variety of treatments employed to stop status epilepticus. Some procedures were clearly inadequate. We recommend the same protocol as that used in generalised convulsive status epilepticus. ${ }^{15}$
We thank Mrs G van Etten for preparing the manuscript

1 Anderman F, Robb J. Absence status. A reappraisal following review of thirty eight patients. Epilepsia 1972;13 177-87.

2 Porter R, Perry J. Petit mal status. In: Delgado-Escueta A, Treiman D, Wasterlain C, Porter R, eds. Advances in neurology, New York: Raven Press, 1983;34:61-9.

3 Guberman A, Cantu-Reyna G, Stuss D, Broughton R Nonconvulsive generalized status epilepticus: clinical features, neuropsychological testing and long-term followup. Neurology 1986;36:1284-91.

4 Gastaut H, Roger J, Roger A. Sur la signifaction de certains fugues epileptiques. $\dot{A}$ propos d'une observation electroclinique d'état de mal temporal. Rev Neurol 1956; 94:298-301.

5 Tomson T, Svanborn E, Wedlund J-E. Nonconvulsive status epilepticus: high incidence of complex partial status. tus epilepticus: high incidenc

6 Tomson T, Lindbom U, Nilsson B. Nonconvulsive status epilepticus in adults: thirty-two consecutive patients from a general hospital population. Epilepsia 1992;33:829-35.

7 Treiman D, Delgado-Escueta A. Complex partial status epilepticus. In: Delgado-Escueta A, Treiman D, Wasterlain C, Porter R, eds. Status epilepticus. Advances in neurology. New York: Raven Press, 1983;34:69-83.

8 Delgado-Escueta A, Treiman D. Focal status epilepticus: modern concepts. In: Lüders H, Lesser R, eds. Epilepsy: electronic syndromes. New York: Springer-Verlag, 1987 347-93.

9 Williamson P, Spencer D, Spencer S, Novelly R, Mattson R. Complex partial status epilepticus: a depth-electrode study. Ann Neurol 1985;18:647-54.

10 Richard P, Brenner R. Absence status: case reports and a review of the literature. L'Encéphale 1980;VI:385-92.

11 Niedermeyer E, Fineyre F, Riley T, Uematsu S. Absence status (petit mal status) with focal characteristics. Arch status (petit mal status)

12 Fagan K, Lee S. Prolonged confusion following convulsions due to generalized nonconvulsive status epilepticus. Neurology 1990;40:1689-94

13 Engel J, Ludwig B, Fetell M. Prolonged partial complex status epilepticus: EEG and behavioral observations. Neurology 1978;28:863-9.

14 Treiman D, Delgado-Escueta A. Complex partial status epilepticus. Fpn f Psychiatry Neurol 1988;42:437-40.

15 Scholtes F, Renier W, Meinardi H. Generalized convulsive status epilepticus. Pathophysiology and treatment. Pharmacy World and Science 1993;15:17-28. 